\title{
Perineural invasion in early-stage cervical cancer and its relevance following surgery
}

\author{
YI ZHU ${ }^{1,2}$, GUONAN ZHANG ${ }^{1,3,4}$, YAN YANG ${ }^{3}$, LING CUI $^{1}$, SHIJUN JIA ${ }^{5}$, \\ YU SHI ${ }^{1}$, SHUIQIN SONG ${ }^{1}$ and SHIQIANG XU ${ }^{1}$
}

\begin{abstract}
Departments of ${ }^{1}$ Gynecological Oncology and ${ }^{2}$ Ultrasound, Sichuan Cancer Hospital \& Institute, Cancer Hospital Affiliated to School of Medicine, University of Electronic Science and Technology of China, Chengdu, Sichuan 610041;

${ }^{3}$ Graduate School, Guangxi Medical University, Nanning, Guangxi Zhuang Autonomous Region 530021;

${ }^{4}$ Department of Gynecology, Chengdu University of Traditional Chinese Medicine, Chengdu, Sichuan 610057;

${ }^{5}$ Department of Pathology, Sichuan Cancer Hospital \& Institute, Cancer Hospital Affiliated to School of Medicine, University of Electronic Science and Technology of China, Chengdu, Sichuan 610041, P.R. China
\end{abstract}

Received April 30, 2016; Accepted April 6, 2017

DOI: $10.3892 / \mathrm{ol} .2018 .8116$

\begin{abstract}
Perineural invasion (PNI) is the neoplastic invasion of nerves by cancer cells, a process that has attracted attention as a novel prognostic factor for cancer. The present study aimed to investigate the prognostic value of PNI in patients with early-stage cervical cancer (International Federation of Gynecology and Obstetrics stage IA2-IIA). A total of 210 patients who underwent radical hysterectomy and pelvic lymphadenectomy between 2007 and 2012 were included in the current study, of whom $8.57 \%$ (18/210) exhibited PNI. Patients with PNI were more likely to exhibit adverse histopathological features, such as increased tumor size, depth of stromal invasion, parametrial invasion, lymphovascular space invasion and lymph nodes metastases (all $\mathrm{P}<0.05$ ). Patients with PNI exhibited shorter disease-free and overall survival $(\mathrm{P}=0.002$ and $\mathrm{P}=0.017$, respectively). However, $\mathrm{PNI}$ was not identified as an independent risk factor for either recurrence or death by multivariate analysis. Furthermore, $88.9 \%(16 / 18)$ of patients with PNI received adjuvant therapy following surgery. PNI was significantly associated with well-established indicators for adjuvant therapy. In conclusion, PNI was associated
\end{abstract}

Correspondence to: Professor Guonan Zhang, Department of Gynecological Oncology, Sichuan Cancer Hospital \& Institute, Cancer Hospital Affiliated to School of Medicine, University of Electronic Science and Technology of China, 55, Section 4, South People's Road, Chengdu, Sichuan 610041, P.R. China

E-mail: zhanggn@hotmail.com

Abbreviations: PNI, perineural invasion; FIGO, International Federation of Gynecology and Obstetrics; RT, radiation therapy; LVSI, lymphovascular space invasion; DFS, disease-free survival; OS, overall survival; NACT, neoadjuvant chemotherapy

Key words: perineural invasion, uterine cervical cancer, prognosis risk factors with multiple high-risk factors and its presence was indicative of a poor outcome in patients with early-stage cervical cancer, which may influence management decisions regarding adjuvant therapy.

\section{Introduction}

Malignant cells dissociate from their environment in the extracellular matrix and establish metastatic deposits at distant sites. The generally recognized route of metastatic spread occurs directly as well as through vascular and lymphatic channels; however, whether tumor cells can spread in and along nerves is not yet widely accepted. Perineural invasion (PNI), also called neurotropic carcinomatous spread or perineural spread, is the neoplastic invasion of nerves, which may prove to be another metastatic route (1). As described in literature since the mid-1800s, PNI has since emerged as a pathological feature of $\sim 90 \%$ of surgical specimens in cancer of the head and neck, prostate, pancreas, colon and rectum; for a number of these malignancies, PNI is independently associated with high recurrence rates and poor survival (2-7).

Early-stage cervical cancer is treated by radical hysterectomy and pelvic lymphadenectomy, with or without sampling of the paraaortic lymph nodes (8). This surgical approach allows for the preservation of ovarian endocrine function and offers greater protection of sexual function. It also makes resection of enlarged lymph nodes possible, and facilitates the histopathological evaluation of the involvement of the parametria and lymph nodes (9). Postoperative adjuvant therapy for cervical cancer should be considered, depending on whether pathological examination reveals the presence of risk factors for disease recurrence (10). Although parametrial and lymph node involvement, and lymphovascular space invasion are well-known poor prognostic indicators in cervical cancer, the histological details of disease status within the parametria and any PNI are often overlooked and rarely reported in histopathological evaluations of radical hysterectomy specimens. The objective of the present study was to investigate the incidence 
of PNI in early-stage (IA2-IIA) cervical cancer and evaluate its prognostic impact.

\section{Materials and methods}

Clinical characteristics of patients. Data from a total of 210 women (median age, 44 years, range 27-72 years) who underwent radical hysterectomy and pelvic lymphadenectomy between January 2007 and December 2012 at the Sichuan Cancer Hospital (Chengdu, China) were assessed in the present study. All patients with International Federation of Gynaecology and Obstetrics (FIGO) stage IA2 to IIA2 squamous cell carcinoma, adenosquamous carcinoma or adenocarcinoma of the cervix were included, with other histological subtypes, such as neuroendocrine or small cell tumors, excluded. Those with incomplete local tumor resection were excluded from the study. Patients with preoperative tumors $>4 \mathrm{~cm}$ underwent platinum-based neoadjuvant chemotherapy (NACT). In total, 134 patients that had at least one high-risk factor or more than two intermediate-risk factors received adjuvant therapy with radiation therapy (RT) and/or 3-7 cycles of concurrent chemoradiation therapy (with a platinum-based regimen) following surgery $(11,12)$. High-risk factors for recurrence of cervical cancer included lymph nodes metastasis, parametrial invasion and resection margin involvement $(11,13)$. Intermediate-risk factors included tumor size, depth of invasion according to the guidelines of Elshawi et al (14), and lymphovascular space invasion (LVSI) $(11,13)$. The recurrence of disease was identified radiologically by computed tomography (CT) or magnetic resonance imaging (MRI). The study was approved by the Research Ethics Committee of the Sichuan Cancer Hospital. Each patient gave written informed consent prior to enrollment in the study.

Histopathological analysis. The pathological features were diagnosed and recorded by two pathologists. PNI was defined as occurring if cancer cells were present in or immediately surrounding nerve bundles, as identified using hematoxylin and eosin staining (15). The tissue in each case was formalin-fixed and embedded in paraffin. Paraffin sections $(5 \mu \mathrm{m}$ thickness) were used for hematoxylin and eosin staining. Table I summarizes patient demographics (age) and tumor features assessed in the study population, including histological subtype, tumor dimensions, depth of invasion, parametrial invasion, lymph nodes metastasis, surgical resection margin status, LVSI and PNI.

Statistical analysis. Disease-free survival (DFS) was defined as the interval between the day that surgery was performed and the day that recurrence was observed radiologically by $\mathrm{CT}$ or MRI. If recurrence was not detected, the date of death or of the last follow-up was used. Overall survival (OS) was defined as the interval between the dates of surgery and death. The follow-up period following the initial operation for the primary lesion was 5 years for DFS and OS. DFS and OS were estimated by Kaplan-Meier curves and were compared using the log-rank test. Time to relapse and death was analyzed using the Cox proportional hazards model for univariate and multivariate analyses. Associations between nominal factors were assessed with $\chi^{2}$ tests, with the application of a

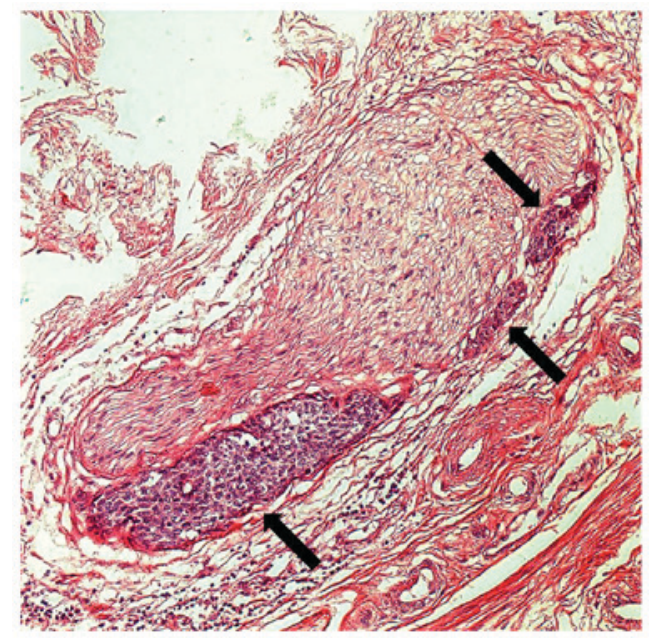

Figure 1. Hematoxylin and eosin-stained histological image of PNI in squamous cell carcinoma of the cervix. PNI of small clusters of the carcinoma is indicated by arrows (magnification, x200). PNI, perineural invasion.

two-tailed Fisher's exact test where appropriate. $\mathrm{P}<0.05$ was considered to indicate statistical significance. Analysis was performed using SPSS 17.0 software (SPSS, Inc., Chicago, IL, USA).

\section{Results}

Cases in which PNI or parametrial invasion was described in the original pathology report were reviewed. A total of $18 / 210$ patients $(8.57 \%)$ presented with PNI; 15 patients exhibited PNI in the cervix, 3 in the cervix and the parametrium. An example of PNI is presented in Fig. 1.

The mean age of subjects with PNI was $47( \pm 7)$ (range 32-62) years compared with $44( \pm 8)$ (range 27-72) years in subjects without PNI $(\mathrm{P}=0.908)$. The association between PNI and known risk factors for recurrence of cervical cancer is presented in Table I. A total of 13 patients with PNI had tumors $>4 \mathrm{~cm}$ vs. 93 subjects without PNI ( $\mathrm{P}=0.045)$. Patients with deeper cervical invasion reported significantly more PNI as those with more superficial invasion $(\mathrm{P}<0.001)$. PNI was significantly associated with parametrial invasion $(\mathrm{P}<0.001)$, lymph node metastasis $(\mathrm{P}=0.001)$ and the increased occurrence of LVSI $(\mathrm{P}=0.005)$. However, there was no significant difference in FIGO stage, tumor histology or extension to the vaginal margin between the two groups (Table I).

Cox proportional hazard regression modeling was performed to determine a predictive association of the clinicopathological variables with recurrence and death-associated with cervical cancer (Table II). At the 5-year follow-up, FIGO stage, tumor size, depth of invasion, parametrial invasion, LVSI, lymph node metastasis and positive vaginal margin were identified to be associated with recurrence by univariate analysis. Depth of invasion, parametrial invasion and positive vaginal margin were risk factors for death in this population (Table II). Models that include all histopathological variables and tumor markers found to have significant prognostic value in univariate analysis were then analyzed by multivariate analysis (Table II). Depth of invasion and parametrial invasion were significantly associated with DFS 
Table I. Histopathological factors assessed in study population.

\begin{tabular}{|c|c|c|c|}
\hline Histopathological factors & Subjects with PNI (n=18) & Subjects without PNI $(\mathrm{n}=192)$ & P-value ${ }^{a}$ \\
\hline Age, years $^{\mathrm{b}}$ & $47.06( \pm 7.78)$ & $44.22( \pm 8.56)$ & 0.908 \\
\hline FIGO stage, n (\%) & & & 0.286 \\
\hline Ia2 & 0 & $3(1.56)$ & \\
\hline Ib1 & $5(27.78)$ & $80(41.67)$ & \\
\hline $\mathrm{Ib} 2$ & $10(55.56)$ & $64(33.33)$ & \\
\hline IIa1 & 0 & $17(8.85)$ & \\
\hline IIa2 & $3(16.67)$ & $28(14.58)$ & \\
\hline Pathology, n (\%) & & & 0.352 \\
\hline $\mathrm{SCC}$ & $16(88.89)$ & $157(81.77)$ & \\
\hline Adeno/adenosquamous carcinoma & $2(11.11)$ & $35(18.23)$ & \\
\hline Histological grade, $\mathrm{n}(\%)$ & & & 0.371 \\
\hline Well differentiated & $2(11.11)$ & $30(15.62)$ & \\
\hline Moderate differentiation & $6(33.33)$ & $88(45.83)$ & \\
\hline Poor differentiation & $10(55.56)$ & $74(38.54)$ & \\
\hline Tumor size & & & 0.045 \\
\hline$\leq 4 \mathrm{~cm}$ & $5(27.78)$ & $99(51.56)$ & \\
\hline$>4 \mathrm{~cm}$ & $13(72.22)$ & $93(48.44)$ & \\
\hline Depth of invasion & & & $<0.001$ \\
\hline$<2 / 3$ & $2(11.11)$ & $111(57.81)$ & \\
\hline$\geq 2 / 3$ & $16(88.89)$ & $81(42.19)$ & \\
\hline Parametrial invasion & & & $<0.001$ \\
\hline Present & 8 & 2 & \\
\hline Absent & 10 & 190 & \\
\hline LVSI & & & 0.005 \\
\hline Present & 7 & 22 & \\
\hline Absent & 11 & 170 & \\
\hline Lymph nodes metastases & & & 0.001 \\
\hline Positive & 10 & 34 & \\
\hline Negative & 8 & 158 & \\
\hline Positive vaginal margin & & & 0.518 \\
\hline Positive & 1 & 7 & \\
\hline Negative & 17 & 185 & \\
\hline
\end{tabular}

${ }^{a}$ Comparison between patients with and without PNI. ${ }^{b}$ Data are presented as the mean \pm standard deviation. PNI, perineural invasion; SCC, squamous cell carcinoma; LVSI, lymphovascular space invasion; FIGO, International Federation of Gynecology and Obstetrics.

and OS (all $\mathrm{P}<0.05)$. Lymph node metastasis was associated with a risk of recurrence only $(\mathrm{P}=0.04$; Table II).

Early-stage cervical cancer patients without PNI had significantly longer DFS times (Fig. 2A) and OS (Fig. 2B) than patients who had evidence of PNI $(\mathrm{P}=0.002$ and $\mathrm{P}=0.017$, respectively). However, PNI was not identified as an independent risk factor for either recurrence [hazard ratio (HR), 0.26; 95\% confidence interval (CI), 0.07-0.91; $\mathrm{P}=0.05]$ or death (HR, 0.28; 95\% CI, 0.05-1.59; $\mathrm{P}=0.15$ ).

The presence of risk factors for recurrence was indicated for postoperative adjuvant treatment. A total of $63.8 \%$ patients (134/210) underwent adjuvant therapy such as RT or concurrent chemoradiation therapy; $88.9 \%$ patients (16/18) with PNI received adjuvant therapy. Patients with
PNI received significantly more adjuvant therapy than those without PNI. PNI was significantly associated with adjuvant therapy ( $88.9 \%$ sensitivity; $62.7 \%$ specificity; odds ratio, 5.0; $\mathrm{P}=0.021)$.

\section{Discussion}

In 1835, PNI was first reported as a notable pathological feature in head and neck cancers that exhibited a predilection for growth along nerves as they made their way toward the intracranial fossa (2). A precise definition of PNI has not yet been established in the literature. In 2009, Liebig et al (16) advocated a broad definition of PNI, characterizing it as a tumor in close proximity to a nerve and involving $\geq 33 \%$ 
Table II. Univariate and multivariate analysis of clinicopathological features and patient survival.

\begin{tabular}{|c|c|c|c|c|c|c|}
\hline \multirow[b]{2}{*}{ Variable } & \multicolumn{3}{|c|}{ 5-year DFS } & \multicolumn{3}{|c|}{ 5-year OS } \\
\hline & HR & $95 \% \mathrm{CI}$ & P-value & HR & $95 \% \mathrm{CI}$ & P-value \\
\hline \multicolumn{7}{|l|}{ Univariate analysis } \\
\hline Age & 1.00 & $0.96-1.04$ & 0.908 & 1.00 & $0.95-1.06$ & 0.868 \\
\hline FIGO stage & 1.43 & $1.06-1.93$ & 0.023 & 1.48 & $0.97-2.26$ & 0.077 \\
\hline Pathology & 1.66 & $0.74-3.69$ & 0.237 & 1.68 & $0.54-5.21$ & 0.392 \\
\hline Histological grade & 1.49 & $0.88-2.51$ & 0.129 & 1.86 & $0.85-4.09$ & 0.104 \\
\hline Tumor size $(\leq 4$ vs. $>4 \mathrm{~cm})$ & 2.71 & $1.26-5.87$ & 0.007 & 1.79 & $0.65-4.93$ & 0.251 \\
\hline Depth of invasion ( $<2 / 3$ vs. $\geq 2 / 3)$ & 5.58 & $2.29-13.56$ & $<0.001$ & 9.12 & $2.07-40.16$ & $<0.001$ \\
\hline Parametrial invasion & 13.79 & $6.13-31.04$ & $<0.001$ & 15.75 & $5.11-48.52$ & $<0.001$ \\
\hline LVSI & 3.31 & $1.51-7.21$ & 0.006 & 1.16 & $0.26-5.16$ & 0.849 \\
\hline Lymph nodes metastases & 4.30 & $2.15-8.60$ & $<0.001$ & 2.59 & $0.94-7.13$ & 0.081 \\
\hline Positive vaginal margin & 2.96 & $0.90-19.72$ & 0.121 & 6.81 & $1.93-23.99$ & $<0.001$ \\
\hline PNI & 3.56 & $1.53-8.29$ & 0.009 & 2.98 & $0.84-10.86$ & 0.049 \\
\hline \multicolumn{7}{|l|}{ Multivariate analysis } \\
\hline FIGO stage & 1.21 & $0.83-1.75$ & 0.324 & & - & - \\
\hline Tumor size $(\leq 4$ vs. $>4 \mathrm{~cm})$ & 1.26 & $0.49-3.26$ & 0.631 & & - & - \\
\hline Depth of invasion ( $<2 / 3$ vs. $\geq 2 / 3)$ & 3.04 & $1.17-7.88$ & 0.022 & 5.85 & $1.23-27.80$ & 0.026 \\
\hline Parametrial invasion & 16.74 & $4.63-60.50$ & $<0.001$ & 19.43 & $4.23-89.28$ & $<0.001$ \\
\hline LVSI & 2.22 & $0.98-5.04$ & 0.057 & & - & - \\
\hline Lymph node metastasis & 2.24 & $1.06-4.75$ & 0.036 & & - & - \\
\hline Positive vaginal margin & - & - & - & 3.14 & $0.78-12.57$ & 0.106 \\
\hline PNI & 0.26 & $0.07-0.91$ & 0.056 & 0.28 & $0.05-1.59$ & 0.154 \\
\hline
\end{tabular}

DFS, disease-free survival; HR, hazard ratio; CI, confidence interval; OS, overall survival; FIGO, International Federation of Gynecology and Obstetrics; PNI, perineural invasion; LVSI, lymphovascular space invasion.

of its circumference or tumor cell invasion in, around and through any of the three layers (the epineurium, perineurium and endoneurium) of the nerve sheath. Various tumor-nerve growth patterns have been described, including complete and incomplete encirclement, concentric lamination and tangential contact (17). A much more frequent finding in PNI is tumor-nerve contact within the perineurum without tumor cells invasion of the sheath (16). A scientific and comprehensive definition of PNI would aid further research and clinical applications.

PNI is associated with pain and tumor metastasis, independent of lymphatic or vascular involvement (18). The mechanism by which PNI occurs remains unclear. For the last 40 years, the predominant theory behind the pathogenesis of PNI has been that cancer cells are marauders that invade nerves through the path of least resistance. However, novel data challenge the conventional theories, suggesting that prostaglandin E2 promotes cancer invasion and, in a positive-feedback mechanism, galanin released by cancer induces neuritogenesis, facilitating PNI (19). PNI may involve reciprocal signaling interactions between tumor cells and nerves, with the invading tumor potentially acquiring the ability to respond to pro-invasive signals within the peripheral nerve milieu. In vitro PNI models of prostate cancer and pancreatic cancer provide evidence for this interaction, suggesting that the signaling mechanisms behind PNI are likely to involve at least three different cellular elements, including tumor cells, nerve cells, and stromal cells, and may include autocrine and paracrine mechanisms $(16,20)$. Other evidence suggests the presence of a complex signaling interaction that involves the exchange of neurotrophic growth factors between nerves and invading tumor cells to drive pathogenesis, factors including neurotropin, granulocyte colony-stimulating factor, and cytokines (21-23). Another study investigated the mechanism of PNI in other aspects, including the role of gene defects and the tumor suppressor TP73 (24).

Patients with early-stage or locally advanced-stage cervical cancer are preferentially treated with radical hysterectomy and pelvic lymph node dissection or NACT followed by radical hysterectomy, achieving 5-year survival rates of 85\% (25-27). Additional adjuvant treatment may be pursued depending on the presence or absence of risk factors for recurrence. Lymph node metastasis, parametrial invasion and resection margin involvement are known to be high-risk factors that increase the recurrence rate up to $40 \%$ in postoperative cervical cancer $(28,29)$. The significance of parametrial resection in the operative management of patients with cervical cancer has led to the evolution of the surgical techniques used to manage disease in these patients. However, thus far, large prognostic studies that deal with morphologic parameters do not even refer to PNI (30-35), and histopathological descriptions of parametria usually ignore the existence of PNI altogether. 

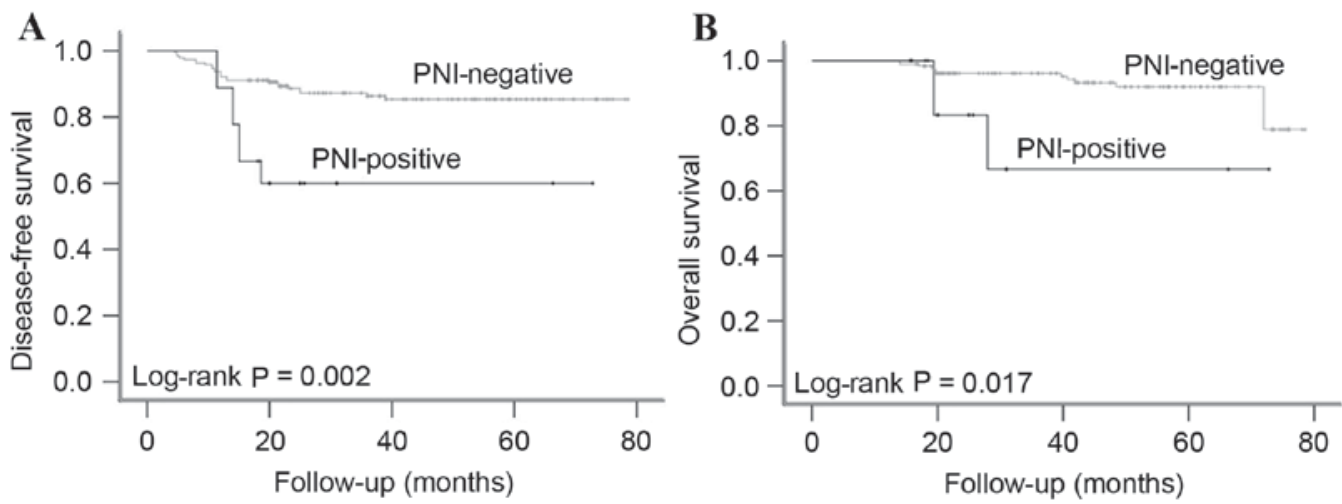

Figure 2. Kaplan-Meier survival curves of DFS and OS in patients with cervical cancer according to PNI expression. (A) The presence of PNI was significantly associated with lower DFS ( $\mathrm{P}=0.002)$. (B) The presence of PNI in cervical cancer was significantly associated with lower OS (P=0.017). 18 subjects with PNI vs. 192 subjects without PNI. DFS, disease-free survival; OS, overall survival; PNI, perineural invasion.

For certain human malignancies, PNI has been found to occur with a high incidence and to be an indicator of poor outcome. However, the knowledge concerning the frequency and the impact of PNI in cervical cancer is limited (14,36-39). In the present study, PNI occurred in $8.57 \%(18 / 210)$ of patients with cervical cancer (FIGO stages IA2-IIA). PNI was detected in the parametrium in only three cases, and in the cervix in all 18 cases. A consensus regarding a standardized definition and reporting of PNI in cervical cancer histopathology reports has not yet been reached. Compared with results of the present study, the incidence of PNI $(8.57 \%, 18 / 210)$ was significantly lower than that reported by Horn et al (38) $(\mathrm{P}=0.002)$. Patients that received NACT therapy were excluded from the present study; thus, NACT may reduce the incidence of PNI.

PNI has been reported to be associated with a variety of well-established risk factors (35-39). In the present study, the presence of PNI was significantly associated with higher FIGO stage, larger tumors $(\leq 4 \mathrm{~cm})$, greater depth of invasion, increased parametrial invasion, LVSI and lymph nodes metastasis $(\mathrm{P}<0.05)$, which are factors that have important roles in deciding adjuvant therapy. The 5-year DFS and OS rates were significantly decreased in early-stage patients presenting with PNI, compared with those that $\operatorname{did} \operatorname{not}(\mathrm{P}=0.002$ and $\mathrm{P}=0.011$, respectively). This indicated that PNI was associated with more aggressive disease; however, when subjected to multivariate analysis, PNI was not found to be an independent high-risk factor that predicted either recurrence (HR, 1.50; 95\% CI, 0.42-5.39) or death (HR, 0.69; 95\% CI, 0.06-8.19). Horn et al (38) reported the 5-year OS rate was significantly decreased in patients with PNI, whereas other studies reported no difference in DFS or OS $(38,39)$. A meta-analysis demonstrated that cervical cancer with PNI was associated with a lower OS rate (40). However, in the present study, association of PNI with more established markers of poor prognosis, or as a particular histological characteristic, was limited, as adjuvant therapy had an impact on OS and DFS. Radiotherapy may be beneficial for patients with PNI and has no marked effect on the quality of life. Fully determining the impact of PNI on the prognosis of patients with cervical cancer requires a large sample study to elucidate further information. In the present study, PNI was demonstrated to be markedly associated with risk factors for recurrence in cervical cancer. Surgeons, radiotherapists and oncologists should therefore be aware of the presence of PNI and should note it in surgical pathology reports.

Nerve-sparing radical hysterectomy (NSRH) has been reported to provide similar cure rates for cervical cancer to strategies using the conventional, non-nerve-sparing technique, with decreased vesical, rectal and, to a lesser degree, sexual dysfunction (41-43). However, the postoperative recurrence rate of patients that undergo this surgical procedure remains unclear and there is no standardized research methodology. There are a number of issues that require clarification by an in-depth study. Since the presence of PNI is associated with risk factors for recurrence in cervical cancer, clinicians may wish to strictly monitor patients who have undergone NSRH. Therefore, the present study set limits on patient eligibility, with patients only eligible if imaging examination revealed no high-risk factors in early-stage patients who did not receive NACT (which may mask the occurrence of PNI). Furthermore, PNI may present in the parametrium in early-stage cervical cancer, so NSRH could retain a risk of recurrence (or residual tumor). PNI can be detected by preoperative imaging studies (CT or MRI) and histological evaluation $(17,44)$. PNI evaluation by 3.0T high-field MRI can map the exact tumor location and improve critical surgical and treatment planning (45). Previous studies have reported that the risk of parametrial involvement is $<1 \%$ for patients with a tumor $<2 \mathrm{~cm}$ in size, limited depth of invasion and no lymphovascular invasion $(46,47)$. This knowledge could be used to aid selection of patients who would most benefit from NSRH without requiring postoperative radiation.

In conclusion, PNI may represent a novel prognostic risk factor for cervical cancer, which is expected to assist in determining patient adjuvant treatment. Further study is required to provide for a deeper insight into the pathogenic mechanisms of PNI. PNI is a novel clinicopathological topic in gynecological oncology and pathology, and required further investigation to aid in reducing disease recurrence and improving DFS and OS in patients with cervical cancer.

\section{Acknowledgements}

Not applicable. 


\section{Funding}

No funding was received.

\section{Availability of data and materials}

The datasets generated and analyzed in the present study are included in this published article.

\section{Authors' contributions}

YZ carried out study design and manuscript editing. GNZ carried out study conception and manuscript review. YY and LC carried out quality control of data and algorithms. SJJ performed data analysis and interpretation, and manuscript preparation. YS carried out statistical analysis. SQS and SQX carried out data acquisition. All authors reviewed the manuscript.

\section{Ethics and consent to participate}

The study was approved by the Research Ethics Committee of the Sichuan Cancer Hospital. Each patient gave written informed consent prior to enrollment in the study.

\section{Consent for publication}

All the study participants gave consent for the data to be published.

\section{Competing interests}

The authors declare that they have no competing interests.

\section{References}

1. Rapidis AD, Givalos N, Gakiopoulou H, Faratzis G Stavrianos SD, Vilos GA, Douzinas EE and Patsouris E: Adenoid cystic carcinoma of the head and neck. Clinicopathological analysis of 23 patients and review of the literature. Oral Oncol 41: 328-335, 2005

2. Cruveilheir J (ed): Maladies Des Nerfs Anatomie Pathologique Du Corps Humain. 2nd edition. Paris, France, JB Bailliere, 1835

3. Ozaki H, Hiraoka T, Mizumoto R, Matsuno S, Matsumoto Y, Nakayama T, Tsunoda T, Suzuki T, Monden M, Saitoh Y, et al: The prognostic significance of lymph node metastasis and intrapancreatic perineural invasion in pancreatic cancer after curative resection. Surg Today 29: 16-22, 1999.

4. Law WL and Chu KW: Anterior resection for rectal cancer with mesorectal excision: A prospective evaluation of 622 patients. Ann Surg 240: 260-268, 2004.

5. Beard CJ, Chen MH, Cote K, Loffredo M, Renshaw AA, Hurwitz M and D'Amico AV: Perineural invasion is associated with increased relapse after external beam radiotherapy for men with low-risk prostate cancer and may be a marker for occult, high-grade cancer. Int J Radiat Oncol Biol Phys 58: 19-24, 2004.

6. Su CH, Tsay SH, Wu CC, Shyr YM, King KL, Lee CH, Lui WY, Liu TJ and P'Eng FK: Factors influencing postoperative morbidity, mortality, and survival after resection for hilar cholangiocarcinoma. Ann Surg 223: 384-394, 1996.

7. Duraker N, Sisman S and Can G: The significance of perineural invasion as a prognostic factor in patients with gastric carcinoma. Surg Today 33: 95-100, 2003.

8. Inoue T: Prognostic significance of the depth of invasion relating to nodal metastases, parametrial extension, and cell types. A study of 628 cases with Stage IB, IIA, and IIB cervical carcinoma. Cancer 54: 3035-3042, 1984.
9. Piver MS, Rutledge F and Smith JP: Five classes of extended hysterectomy for women with cervical cancer. Obstet Gynecol 44: 265-272, 1974.

10. Lai CH, Hong JH, Hsueh S, Ng KK, Chang TC, Tseng CJ, Chou HH and Huang KG: Preoperative prognostic variables and the impact of postoperative adjuvant therapy on the outcomes of Stage IB or II cervical carcinoma patients with or without pelvic lymph node metastases: An analysis of 891 cases. Cancer 85: 1537-1546, 1999.

11. Delgado G, Bundy B, Zaino R, Sevin BU, Creasman WT and Major F: Prospective surgical-pathological study of disease-free interval in patients with stage IB squamous cell carcinoma of the cervix: A gynecologic oncology group study. Gynecol Oncol 38: 352-357, 1990.

12. Rotman M, Sedlis A, Piedmonte MR, Bundy B, Lentz SS, Muderspach LI and Zaino RJ: A phase III randomized trial of postoperative pelvic irradiation in Stage IB cervical carcinoma with poor prognostic features: Follow-up of a gynecologic oncology group study. Int J Radiat Oncol Biol Phys 65: 169-176, 2006.

13. Ho CM, Chien TY, Huang SH, Wu CJ, Shih BY and Chang SC: Multivariate analysis of the prognostic factors and outcomes in early cervical cancer patients undergoing radical hysterectomy. Gynecol Oncol 93: 458-464, 2004.

14. Elsahwi KS, Barber E, Illuzzi J, Buza N, Ratner E, Silasi DA, Santin AD, Azodi M, Schwartz PE and Rutherford TJ: The significance of perineural invasion in early-stage cervical cancer. Gynecol Oncol 123: 561-564, 2011.

15. Marchesi F, Piemonti L, Mantovani A and Allavena P: Molecular mechanisms of perineural invasion, a forgotten pathway of dissemination and metastasis. Cytokine Growth Factor Rev 21: 77-82, 2010.

16. Liebig C, Ayala G, Wilks JA, Berger DH and Albo D: Perineural invasion in cancer: A review of the literature. Cancer 115: 3379-3391, 2009.

17. Fagan JJ, Collins B, Barnes L, D'Amico F, Myers EN and Johnson JT: Perineural invasion in squamous cell carcinoma of the head and neck. Arch Otolaryngol Head Neck Surg 124: 637-640, 1998 .

18. Binmadi NO and Basile JR: Perineural invasion in oral squamous cell carcinoma: A discussion of significance and review of the literature. Oral Oncol 47: 1005-1010, 2011.

19. Scanlon CS, Banerjee R, Inglehart RC, Liu M, Russo N, Hariharan A, van Tubergen EA, Corson SL, Asangani IA, Mistretta CM, et al: Galanin modulates the neural niche to favour perineural invasion in head and neck cancer. Nat Commun 6: 6885, 2015.

20. Panizza B, Warren TA, Solares CA, Boyle GM, Lambie D and Brown I: Histopathological features of clinical perineural invasion of cutaneous squamous cell carcinoma of the head and neck and the potential implications for treatment. Head Neck 36: 1611-1618, 2014.

21. Ketterer K, Rao S, Friess H, Weiss J, Büchler MW and Korc M: Reverse transcription-PCR analysis of laser-captured cells points to potential paracrine and autocrine actions of neurotrophins in pancreatic cancer. Clin Cancer Res 9: 5127-5136, 2003.

22. Marchesi F, Piemonti L, Fedele G, Destro A, Roncalli M, Albarello L, Doglioni C, Anselmo A, Doni A, Bianchi P, et al: The chemokine receptor CX3CR1 is involved in the neural tropism and malignant behavior of pancreatic ductal adenocarcinoma. Cancer Res 68: 9060-9069, 2008.

23. Schweizerhof M, Stösser S, Kurejova M, Njoo C, Gangadharan V, Agarwal N, Schmelz M, Bali KK, Michalski CW, Brugger S, et al: Hematopoietic colony-stimulating factors mediate tumor-nerve interactions and bone cancer pain. Nat Med 15: 802-807, 2009.

24. Prueitt RL, Yi M, Hudson RS, Wallace TA, Howe TM, Yfantis HG, Lee DH, Stephens RM, Liu CG, Calin GA, et al: Expression of microRNAs and protein-coding genes associated with perineural invasion in prostate cancer. Prostate 68: 1152-1164, 2008.

25. Sevin BU,Lu Y,Bloch DA, Nadji M, Koechli OR and Averette HE: Surgically defined prognostic parameters in patients with early cervical carcinoma. A multivariate survival tree analysis. Cancer 78: 1438-1446, 1996.

26. Buda A, Fossati R, Colombo N, Fei F, Floriani I, Gueli Alletti D, Katsaros D, Landoni F, Lissoni A, Malzoni C, et al: Randomized trial of neoadjuvant chemotherapy comparing paclitaxel, ifosfamide, and cisplatin with ifosfamide and cisplatin followed by radical surgery in patients with locally advanced squamous cell cervical carcinoma: The SNAP01 (Studio Neo-Adjuvante Portio) Italian collaborative study. J Clin Oncol 23: 4137-4145, 2005. 
27. Vizza E, Pellegrino A, Milani R, Fruscio R, Baiocco E, Cognetti F, Savarese A, Tomao F, Chen C and Corrado G: Total laparoscopic radical hysterectomy and pelvic lymphadenectomy in locally advanced stage IB2-IIB cervical cancer patients after neoadjuvant chemotherapy. Eur J Surg Oncol 37: 364-369, 2011

28. Lahousen M, Haas J, Pickel H, Hackl A, Kurz C, Ogris H, Stummvoll W and Winter R: Chemotherapy versus radiotherapy versus observation for high-risk cervical carcinoma after radical hysterectomy: A randomized, prospective, multicenter trial. Gynecol Oncol 73: 196-201, 1999.

29. Rose PG: Advances in the management of cervical cancer. J Reprod Med 45: 971-978, 2000.

30. Morice P, Castaigne D, Pautier P, Rey A, Haie-Meder C, Leblanc $M$ and Duvillard P: Interest of pelvic and paraaortic lymphadenectomy in patients with stage IB and II cervical carcinoma. Gynecol Oncol 73: 106-110, 1999.

31. Pieterse QD, Kenter GG, Eilers PH and Trimbos JB: An individual prediction of the future (disease-free) survival of patients with a history of early-stage cervical cancer, multistate model. Int J Gynecol Cancer 18: 432-438, 2008.

32. Sartori E, Tisi G, Chiudinelli F, La Face B, Franzini R and Pecorelli S: Early stage cervical cancer: Adjuvant treatment in negative lymph node cases. Gynecol Oncol 107 (1 Suppl 1): S170-S174, 2007.

33. Singh N and Arif S: Histopathologic parameters of prognosis in cervical cancer-a review. Int J Gynecol Cancer 14: 741-750, 2004.

34. Takeda N, Sakuragi N, Takeda M, Okamoto K, Kuwabara M, Negishi H, Oikawa M, Yamamoto R, Yamada H and Fujimoto S: Multivariate analysis of histopathologic prognostic factors for invasive cervical cancer treated with radical hysterectomy and systematic retroperitoneal lymphadenectomy. Acta Obstet Gynecol Scand 81: 1144-1151, 2002.

35. Trimbos JB, Lambeek AF, Peters AA, Wolterbeek R, Gaarenstroom KN, Fleuren GJ and Kenter GG: Prognostic difference of surgical treatment of exophytic versus barrel-shaped bulky cervical cancer. Gynecol Oncol 95: 77-81, 2004.

36. Memarzadeh S, Natarajan S, Dandade DP, Ostrzega N, Saber PA Busuttil A, Lentz SE and Berek JS: Lymphovascular and perineural invasion in the parametria: A prognostic factor for early-stage cervical cancer. Obstet Gynecol 102: 612-619, 2003.

37. Ozan H, Ozuysal S and Ediz B: Perineural invasion in early-stage cervical carcinoma. Eur J Gynaecol Oncol 30: 379-383, 2009.
38. Horn LC, Meinel A, Fischer U, Bilek K and Hentschel B: Perineural invasion in carcinoma of the cervix uteri-prognostic impact. J Cancer Res Clin Oncol 136: 1557-1562, 2010.

39. Cho HC, Kim H, Cho HY, Kim K, No JH and Kim YB: Prognostic significance of perineural invasion in cervical cancer. Int J Gynecol Pathol 32: 228-233, 2013.

40. Cui L, Shi Y and Zhang GN: Perineural invasion as a prognostic factor for cervical cancer: A systematic review and meta-analysis. Arch Gynecol Obstet 292: 13-19, 2015.

41. Sakamoto S and Takizawa K: An improved radical hysterectomy with fewer urological complications and with no loss of therapeutic results for invasive cervical cancer. Baillieres Clin Obstet Gynaecol 2: 953-962, 1988.

42. van den Tillaart SA, Kenter GG, Peters AA, Dekker FW, Gaarenstroom KN, Fleuren GJ and Trimbos JB: Nerve-sparing radical hysterectomy: Local recurrence rate, feasibility, and safety in cervical cancer patients stage IA to IIA. Int J Gynecol Cancer 19: 39-45, 2009.

43. Cibula D, Velechovska P, Sláma J, Fischerova D, Pinkavova I, Pavlista D, Dundr P, Hill M, Freitag P and Zikan M: Late morbidity following nerve-sparing radical hysterectomy. Gynecol Oncol 116: 506-511, 2010.

44. Katz B, Srougi M, Dall'Oglio M, Nesrallah AJ, Sant'anna AC, Pontes J Jr, Antunes AA, Reis ST, Viana N, Sañudo A, et al: Perineural invasion detection in prostate biopsy is related to recurrence-free survival in patients submitted to radical prostatectomy. Urol Oncol 31: 175-179, 2013.

45. Penn R, Abemayor E, Nabili V, Bhuta S and Kirsch C: Perineural invasion detected by high-field 3.0-T magnetic resonance imaging. Am J Otolaryngol 31: 482-484, 2010.

46. Reade CJ, Eiriksson LR and Covens A: Surgery for early stage cervical cancer: How radical should it be? Gynecol Oncol 131: 222-230, 2013

47. Basaran D, Dusek L, Majek O and Cibula D: Oncological outcomes of nerve-sparing radical hysterectomy for cervical cancer: A systematic review. Ann Surg Oncol 22: 3033-3040, 2015. 